\title{
ID Numbers Recognition by Local Similarity Voting
}

\author{
Shen $\mathrm{Lu}^{1}$, Yanyun $\mathrm{Qu}^{2, *}$, Yanyun $\mathrm{Cheng}^{3}, \mathrm{Yi} \mathrm{Xie}^{4}$ \\ Xiamen University \\ Computer Science Department \\ Xiamen, China
}

\begin{abstract}
This paper aims to recognize ID numbers from three types of valid identification documents in China: the firstgeneration ID card, the second-generation ID card and the driver license of motor vehicle. We have proposed an approach using local similarity voting to automatically recognize ID numbers. Firstly, we extract the candidate region which contains ID numbers and then locate the numbers and characters. Secondly, we recognize the numbers by an improved template matching method based on the local similarity voting. Finally, we verify the ID numbers and characters. We have applied the proposed approach to a set of about 100 images which are shot by conventional digital cameras. The experimental results have demonstrated that this approach is efficient and is robust to the change of illumination and rotation. The recognition accuracy is up to $98 \%$.
\end{abstract}

Keywords-template matching algorithm; ID number recognition; OCR

\section{INTRODUCTION}

The admission control of private or safety regions has been widely used nowadays. A person is required to show his valid identification document when he enters airports, hotels, and railway stations, etc. In China, there are three main types of valid identification documents: the first-generation ID card, the second-generation ID card and the driver license of motor vehicle. If all identification documents are checked manually, it will be laborious and monotonous for officers/administers. Furthermore, along with the day-by-day distensible human contacts, the needs of valid identification documents inspection will grow; however, just depending on the manual control may lead to lower efficiency. So it is necessary to implement the automatic checking of valid identification documents. In this paper, we aim to recognize the ID numbers in the three types of documents mentioned above.

These identification documents have different layout, as shown in Table I. There are 15 digits, or 18 digits, or 17 digits along with a verification letter in the first-generation ID card, and the face image is in the left of the card. There are 18 digits, or 17 digits along with a verification letter in the secondgeneration ID card, and the face image is in the right of the card. And there are 15 digits, 18 digits, or 17 digits along with a verification letter in the driver license, and the face image is

The research work was supported by the National Basic Research Program of China under Grant No. 2007CB311005, the Fundamental Research Funds for the Central Universities, No.2010121067, and National Defense Basic Scientific Research program of China under Grant B1420110155. the right bottom of the driver license. When referring to the positions of the ID numbers, they are both at the bottom of the two ID cards, and are in the middle of the driver license.

In order to deal with these three layouts, we need to solve three difficulties. The first is to locate ID numbers. The second is to recognize the ID numbers considering the impacts of illumination, rotation and blur. The third is to implement the real-time processing.

TABLE I. ThE MAIN LAYOUT IN ID DOCUMENTS

\begin{tabular}{|c|l|l|l|}
\hline $\begin{array}{c}\text { First-generation } \\
\text { ID Card } \\
\text { of Face Image }\end{array}$ & $\begin{array}{l}\text { In the left of } \\
\text { the card }\end{array}$ & $\begin{array}{l}\text { At the bottom of } \\
\text { the card }\end{array}$ & $\begin{array}{l}15 \text { digits; } \\
\text { or } 18 \text { digits; } \\
\text { or } 17 \text { digits }+1 \text { letter }\end{array}$ \\
\hline $\begin{array}{c}\text { Second-generation } \\
\text { ID Card }\end{array}$ & $\begin{array}{l}\text { In the right of } \\
\text { the card }\end{array}$ & $\begin{array}{l}\text { At the bottom of } \\
\text { the card }\end{array}$ & $\begin{array}{l}18 \text { digits; } \\
\text { or } 17 \text { digits }+1 \text { letter }\end{array}$ \\
\hline Driver License & $\begin{array}{l}\text { In the right } \\
\text { bottom of the } \\
\text { driver license }\end{array}$ & $\begin{array}{l}\text { In the middle of } \\
\text { the driver license }\end{array}$ & $\begin{array}{l}15 \text { digits; } \\
\text { or } 18 \text { digits; } \\
\text { or } 17 \text { digits }+1 \text { letter }\end{array}$ \\
\hline
\end{tabular}

Optical Character Recognition (OCR) is an important branch in image recognition. There are many works to introduce handwriting recognition. Suen [1] surveyed the handwriting problem before. And the neural network was widely used to solve handwriting recognition [2, 3]. But classical methods based on neural network are time-consuming in training stage. Tappert et al. [4] proposed an on-line approach for handwriting recognition. After that, digital number recognition became an active research problem [5-10] because it was nearer to the industrial application.

However, the existing works are not suitable to our scenario, because the digital numbers are mainly standard printed in the ID card, which may simplify our problem. We design a target-oriented systematic approach which can avoid the complicated middle computation. We focus on making our approach embedded in hardware devices which require our approach to be fast and efficient in computation. The proposed approach of ID number recognition is based on the similarity voting. Unlike the prior works, the proposed approach need not train a lot of data beforehand. Our experiments demonstrate that the recognition accuracy achieves $98 \%$ and the speed is less 1 second per image. 
The rest of this paper is organized as follows: In section 2, we briefly discuss related works. Then we describe the extraction and recognition of ID numbers in section 3 and systematically evaluate our method in section 4. Finally, we draw conclusions.

\section{RELATED WORKS}

OCR aims at making computer recognize text characters. Recently, the automatic identification technology for printed and handwritten numbers and characters has been applied in many fields. Especially, the technology for the ID number recognition gets more attention because of its wide applications.

A few years ago, most researchers paid attentions to locating the characters in the first generation ID card [15, 17] and segmenting the ID numbers $[14,16]$. After that, the researchers rushed to develop new recognition system for the second generation ID card [12, 13]. However, the existing ID number identification systems have several disadvantages as follows:

- All images are required to be gotten by scanners or CCD cameras under the interior light. However, these two devices are expensive and not popular which limits the application of these systems.

- Most existing systems almost just pay attention to the second-generation ID card, while in China there are three types of valid identification documents.

- The major technologies used in digital recognition are the BP neural network and the geometric point features based on the digital Hilditch thinning. However, both technologies are rarely used in real applications because of the high complexity and the low efficiency.

In this paper, we design a systematic approach and develop a system to deal with the images shot by conventional digital cameras, and our approach is robust to blur, rotation, illumination and over-exposure. And besides the secondgeneration ID card, the system can identify the ID numbers from the first-generation ID cards and the driving licenses automatically. The proposed approach is a simple but efficient local-similarity-voting method, which combines an improved template matching algorithm with the structure features of digital numbers. Many experiments have verified that it can achieve a high rate of accuracy.

\section{THE FRAMEWORK OF OUR APPROACH}

The framework of our approach is shown in Fig.1. There are mainly four parts in our system: firstly, we detect the face image; secondly, we estimate the ID numbers region; thirdly, we recognize the ID numbers; finally, we verify and correct the ID numbers and output the result.

Because the layout of the three types of documents is different, it is important to ascertain the type of the query image. We use a simple but efficient method to acquire the type of a query image. We observe that the face image is a salient feature for the three documents. The face image locates in the left of the first-generation ID card, and in the right of the second-generation ID card, and in the right bottom of the driver license. So we can ascertain the type of the query image according to the location of face image. We detail our approach in the following.

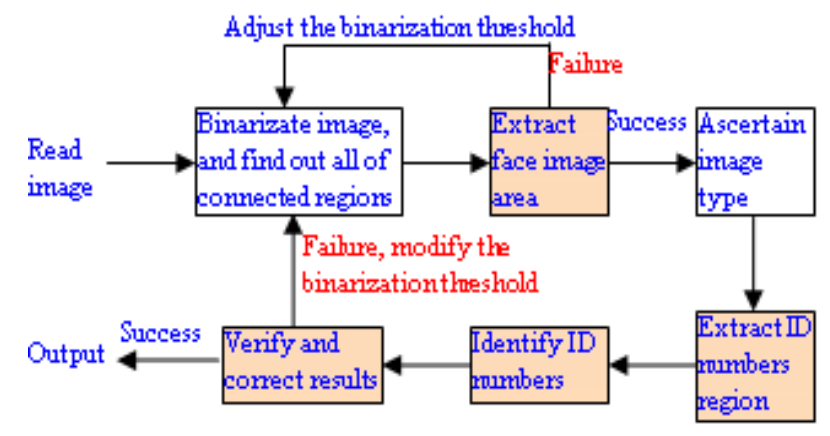

Figure 1. The framework of our approach.

\section{A. Face Localization}

Considering that the human face occupy the most remarkable position in the ID cards and the driver license, we use an adaptive threshold to extract the human face area, and then ascertain the image type according to the location of the human face.

We define the pixels between 180 and 255 gray level as "bright pixels". If the bright pixels in the document image reach to $5 \%$, the image belongs to "bright" images; otherwise, it is a "dark" image. The initial binarization threshold is set as 100 for the bright images and 70 for the dark images (both threshold values are acquired by experience).

We find that when the binarization threshold value is suitable, the face image usually forms the largest connected regions in the binary images: (1) in the first-generation ID card, the hair, the collar, and the five-pointed star regions are universally most conspicuous in the whole binary picture; (2) in the second-generation ID card, the hair, the collar or the entire head regions usually are the largest connected regions; (3) in the driver license, the hair, the collars, the seals or the whole head regions usually occupy most conspicuously; as shown in Fig.2. Therefore, the binarization threshold value can be adaptively decided by the ratio between the connected region area and the document region area. We search the connected regions in the query image, and analyze the ratio between the largest connected region area in the left side to the one in the right side. Furthermore, we extract the face region according to the location of the largest connected region. Finally, we ascertain which type the query image belongs to. The details of the judging rule are as follows:

- If the ratio between the area of left-side largest connected region to the right-side one is under 0.5 , the image may be a second-generation ID card or a driver license. In this case, in order to decide the ID card type, we need to associate with the location of the largest connected region in the document region: if the largest connected region is in the right of the query image, it is a second-generation ID card; if the largest connected region is in the right bottom of the query image, it is a driver license. 
- If the ratio between the left-side largest connected region area to the right-side one is above 1.0, and the image has been judged as neither a driver license nor a second-generation ID card, that is, the ratio is not conformed to the two conditions mentioned above, and then it is a first-generation ID card.
We do not use the Otsu's method in [11] to modify the binarization threshold, because the images shot by conventional cameras are often noisy and influenced by illumination changes. Moreover, in the three types of documents, the color information is different. The Otsu's method does not obtain the expected results.

- If the ratio between the left-side largest connected region area to the right-side one is above 0.75 under 2.5 , the image is a driver license.

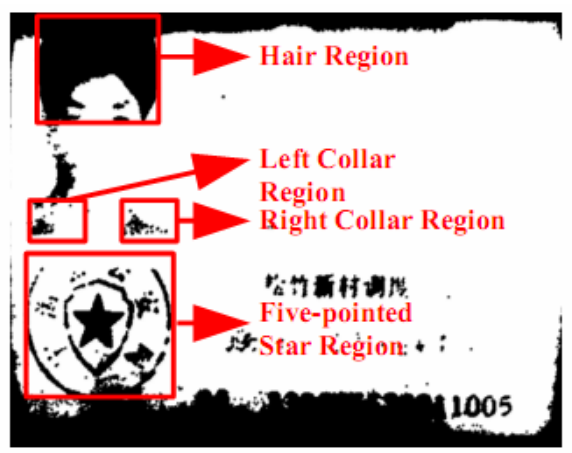

(a) (b)

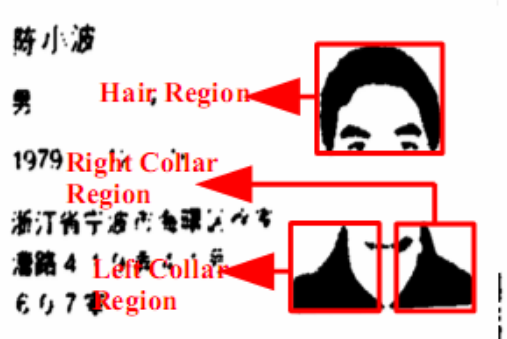

330227197912164710

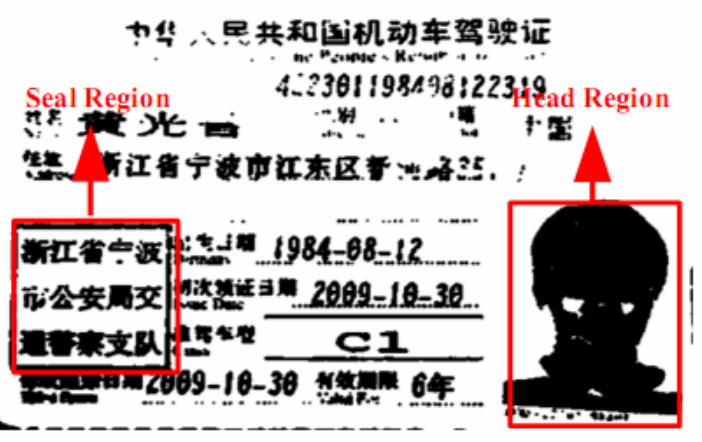

(c)

Figure 2. The examples of binary images: (a) the first generation ID card; (b) the second generation ID card; (c) the driver license.

\section{B. ID Number Localization}

According to the face location, we can ascertain the image type, and then locate the ID numbers region. In the firstgeneration ID card and the second-generation ID card, the ID numbers are all in the bottom of the image, while they are in the middle of the driver license. However, there are two inevitable problems in ID numbers region detection, one is the illumination and the other is the rotation. In the following, we detail our approach on dealing with the illumination and the rotation.

1) Special details about illumination: Variations in the ambient illumination pose a significant challenge to ID numbers recognition algorithms. We propose a simple method to compensate such variations and boost recognition performance. Illumination variations cause intensity changes among each point and its neighbors. We modify the binarization threshold iteratively which is increased gradually for the bright images, or is lowered gradually for the dark images, until the ratio of the black pixels in the literalness region reached above $1 / 6$ but under $1 / 3$. Fig 3(a) shows the initial image which is the middle result after face localization stage. Its black pixels distribute very little in the literalness region, so we increase its binarization threshold, and Fig 3(b) shows the result after the first threshold is modified. But the ratio of the black pixels is still under $1 / 6$, we have to modify the threshold iteratively, until the ratio of the black pixels reach $1 / 6$ or above. Fig 3(c) shows the middle result after the threshold value is modified 4 times, and Fig 3(d) shows the final result image. We can observe that the literalness region is restored sequentially.

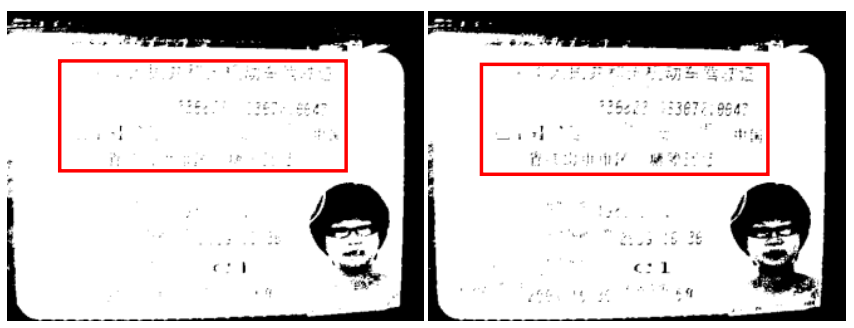

(a) (b)

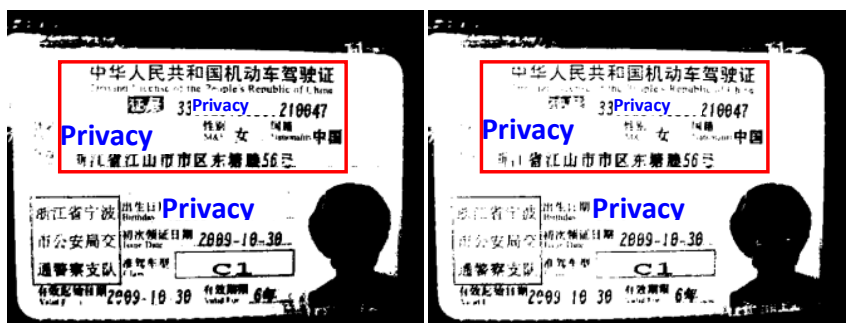

(b) (d)

Figure 3. Schematic diagrams for illumination image. The red box area is the threshold adjustment region. (a) Initial binary image; (b) After first threshold modified; (c) After 4th-time threshold modified; (d) Final binary image

2) Special details about rotation: Image distortion caused by rotation is another challenge to ID numbers recognition. Considering that the face image is a salient feature in the ID card, we find the baseline based on the key points and compute 
the rotation angle. As shown in Fig 4, there are two pairs of the key points in the face, one is the pair of collar-end points, and the other is the pair of temples. In an upright image, each pair of key points ought to lie on a horizontal line. So we firstly detect the two collar-end points by extracting the collar connected region, and then compute the angle between the line passing through the two points and the horizontal line. We use the two temples to verify the rotation angle.

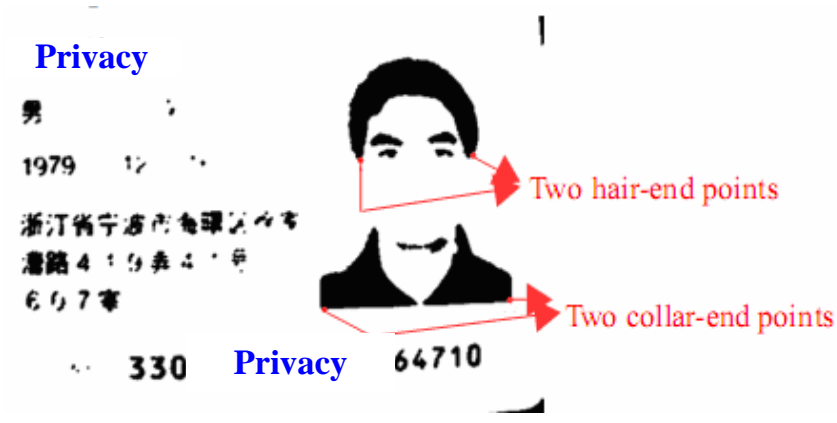

Figure 4. Baseline detection based on pairs of key points.

\section{ID Number Extraction}

Since the ID numbers region contains more than one character, in order to recognize the character, we must segment each character. We use the projection profile to solve the problem. As shown in Fig.5, we firstly transform the ID numbers region to a binary image, and then compute its horizontal and vertical histogram, finally segment each character.

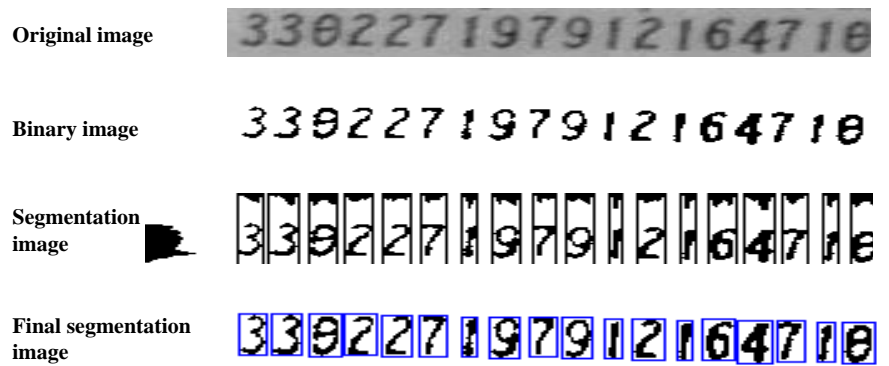

Figure 5. An example for histogram segmentation.

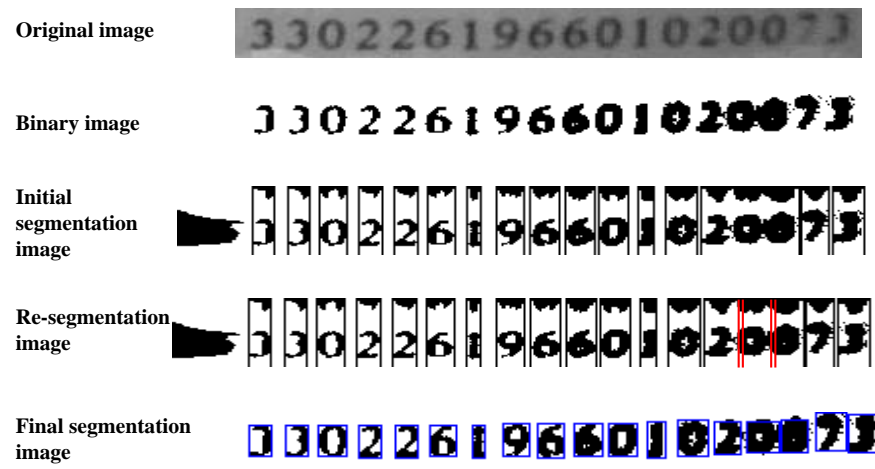

Figure 6. An example for adhesion segmentation.
Number adhesion is another challenge for us, yet we find that the aspect ratio of a single character region is about $1 / 2$. So if the aspect ratio exceeded $1 / 2$, there is adhesion between the characters. We gradually modify the binarization threshold until all the characters have been segmented distinctly. The processing is shown in Fig. 6.

\section{ID Numbers Recognization}

Among the recognition algorithms, the template matching algorithm is undoubtedly popular. It measures the similarity between the templates and the query image, and the query image belongs to the character whose template has the largest similarity value. Obviously, the template matching is based on the characters' geometrical features. Take two-dimensional image for an example, we briefly introduce the matching algorithm.

Firstly, we search the matching pair points between the query image and the template. Let $p$ denote the point in the template and $p^{\prime}$ denote the point in the query image; if the grayscale value of $p$ is equal to that of $p^{\prime}$, the two points are matched. We count the number of the matching points as the similarity score. Let $f(x, y)$ be the query image, and $t(x, y)$ be the template, and $r$ be the matching score. We define the foreground as 1 and the background as 0 , therefore the similarity score is computed as

$$
r=\frac{\sum f(x, y) \cdot t(x, y)}{\sum t(x, y)}
$$

and the related characters have the greater similarity value. Due to the difference of the characters' fonts in the three types of identification documents, we adopted three sets of different font templates for matching. Fig.7 displays all of the templates we used, and the size of the template is $30 \times 45$.

Figure 7. The templates in three types of fonts: (a) the font templates of first-

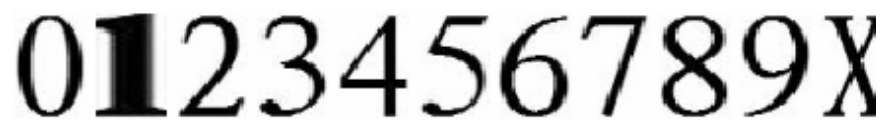

(a)

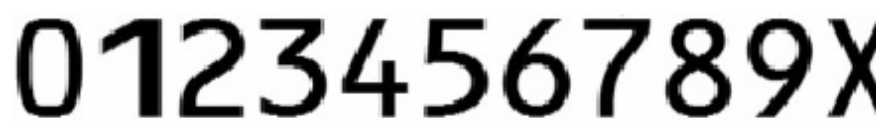

(b)

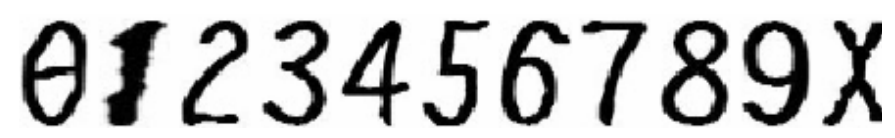

(c)

generation ID cards;(b) the font templates of second-generation ID cards; (c) the font templates of driving licenses.

However, the classical template matching algorithm is susceptible to noise, and the conventional digital camera may make noise. In order to improve the recognition accuracy, we propose a novel template matching method which is based on similarity voting. And we compute the similarity score not 
comparing the point pair between the template and the query image, but based on the local patch.

Take the point $\mathrm{p}$ in the binary standard template for example, as shown in Fig. 8, we search its corresponding point $\mathrm{p}^{\prime}$ in the query image. We judge whether there is a point whose value is equal to that of the point $\mathrm{p}^{\prime}$ in the neighborhood of the point p'. And we set a counter for each standard template. If the point exists, the counter adds one. Followed by the operation, we search for each point of the template in the query image, and the amount of the successful matching points is used to compute the similarity score as

$$
r=\frac{\sum_{i=2}^{n-1} \sum_{j=2}^{m-1} \sigma\left(\sum_{p=i-k}^{i+k} \sum_{q=j-k}^{j+k} f(i, j) \cdot t(p, q)\right)}{(n-2) \cdot(m-2)}
$$

where $\mathrm{n}$ and $\mathrm{m}$ denote the width and length of the template; $\mathrm{k}$ denotes the size of searching window, in our experiments the size of searching windows is $3 \times 3$, and the function $\sigma(\cdot)$ is defined as

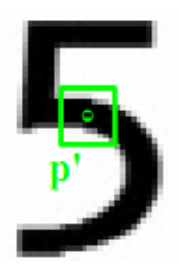

(a) (b)

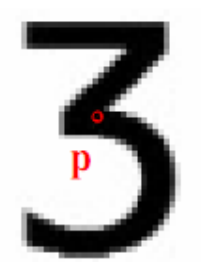

(c)

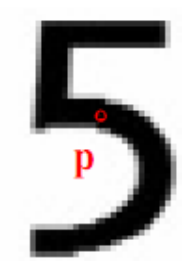

$$
\sigma(x)= \begin{cases}1, & x>0 \\ 0, & x \leq 0\end{cases}
$$

Figure 8. An example for the improved template matching algorithm. (a) The input image; (b) Template of the digit 3; (c) Template of the digit 5.

\section{E. Improved Template Matching Algorithm with the Structure Features of a Character}

In order to improve the recognition accuracy, we propose to combine the structure features of a character with the improved template matching algorithm, because the structure features of a character are different from each other. Take the digit "5" in the second-generation ID card for an example, as shown in Fig.9, if we draw a vertical line at the half width of the template, we will get six intersections. If we draw a horizontal line at a quarter height of the template, we will get two intersections, which are both on the left side of the template. If we draw a horizontal line at $2 / 3$ height of the image, we will obtain two intersections which are both on the right side of the template. We select the distinctive rules for each digital in order to further verify the query image. To the matched candidate templates which pass the verification, we increase their voting weights; and to the matched candidate templates which do not pass the verification, we lower their voting weights. Then we choose the digital template which has the largest voting weight as the new matched template.

The method further improves the recognition accuracy, especially for the low-resolution images, rotated images, as well as the images which has larger projective transformation.

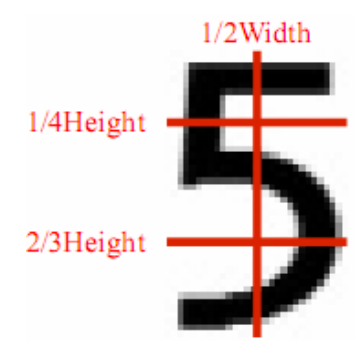

Figure 9. The structure features of number " 5 "

\section{F. ID Number Verfication}

As we know there are some rules designed for the ID number. For the 18-bit ID numbers, the first 6 digits stand for an area code, the digits from the 7th to 14th bit stand for the birthday of a person, the digits from 15 th to 17 th bit stand for the born order on the same day, and the 18th bit stand for a check code. The last character of the ID number can be computed according to the check code rules. Moreover, for the 15-bit ID numbers, the first 6 digits stand for an area code, the digits from 7 th to 12 th bit stand for the birthday of a person, and the 13th to 15th bit stand for the born order on the same day. We can correct the recognition result with the help of those rules mentioned above. Therefore, we save two other candidate numbers for each ID bit. The three candidate templates are of the largest weights. We verify whether the recognition result is satisfied the arrangement rules of ID number, if the verification is successful, we output the recognition result; otherwise, we alternately replace each bit with its candidate template, and until the verification is successful. The final recognition result is the candidate ID number with the largest similarity sum of the 18 or 15 bits. If there are not any successful candidate template for a bit, we modify the binarization threshold of the whole image and start our operation again.

For an example, in the recognition stage we obtain an ID code: 330226196601020013 , but the ID code is not satisfied with the arrangement rules of 18-bit ID numbers, and we find in the verification stage that it is a mistake. We use the two other candidate digits one by one to replace the current bit. We find that the ID number is successful to verification when the digit 1 in the 17th bit is replaced with the digit 7, and it has the largest similarity sum in all of the alterative codes that could successfully pass the information verification test, so we choose it as the final recognition result.

The verification stage can improve the recognition accuracy further.

\section{EXPERIMENTAL RESULTS}

We implement our approach on a dataset which has about one hundred collected ID card images. The system is supported by VC6.0++, and is running on PIII1.8G with 512MB memory. The running time is about 1000 milliseconds average for each 
query ID image. Table II shows some recognition results, and they demonstrate that our approach is not only successful to recognize the ID number in the good images, but also robust for the variation of image rotation, blurring and illumination and the low-resolution image.

Considering that the ID number is a personal privacy, we do not show the whole result of each ID number, and the part of ID number in the original image is occluded. So the star is corresponding to the occluded digits.

In order to evaluate the effect of the structure features of a character, we compare template matching algorithm with the structure features and the one without the structure features.Fig.10 demonstrates the recognition accuracy of the both two configurations. The recognition accuracy for the approach with the structure features is $98.51 \%$, and the one for the approach without the structure features is $94.55 \%$, the structure features can improve the accuracy of the recognition by about $4 \%$.

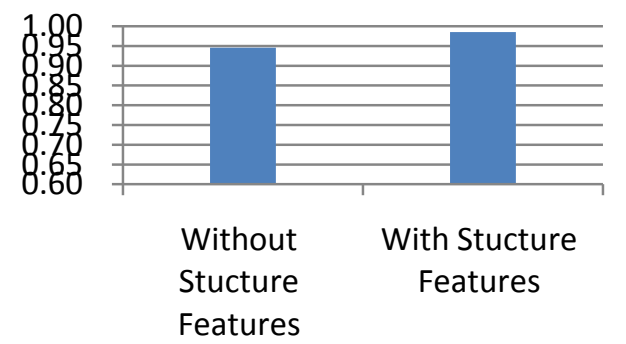

Figure 10. Improvement of recognition with structure features

In order to evaluate the performance of our approach, we also compare our method with OCR engine. Currently, there is rarely OCR engine aiming at recognizing digits only. We use a popular OCR engine named Tesseract for comparison. Tesseract was one of the top 3 engines in the 1995 UNLV Accuracy test. Even to the present, it is probably one of the most accurate open source OCR engines. The source code is available on Google Code [18]. As the OCR engine required pure text image as input, we firstly extract the ID areas from the ID card images. In the ID regions, Otsu's method is used for binarization and the resulting binary images are used as the input of the OCR engine. We compare our method with OCR according to the recognition precision and the average time of processing the recognition ID number except the ID number detection.

As shown in Fig. 11, the precision of our approach achieve $98.51 \%$, which is much higher than the Tesseract OCR engine. Average processing time of proposed method to recognize ID numbers is $45 \mathrm{~ms}$, which is almost 4 times faster than the Tesseract OCR engine.

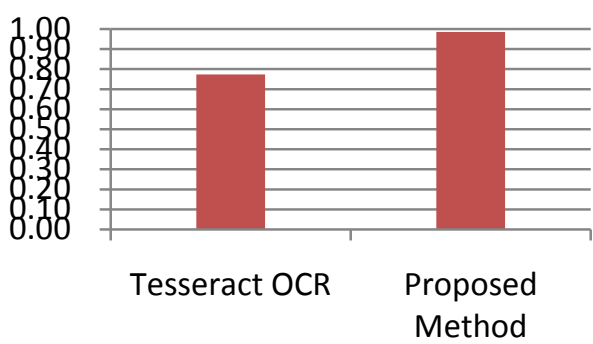

Figure 11. Comparison of Tesseract OCR with the proposed method

TABLE II. SOME RECOGNITON RESUlTS

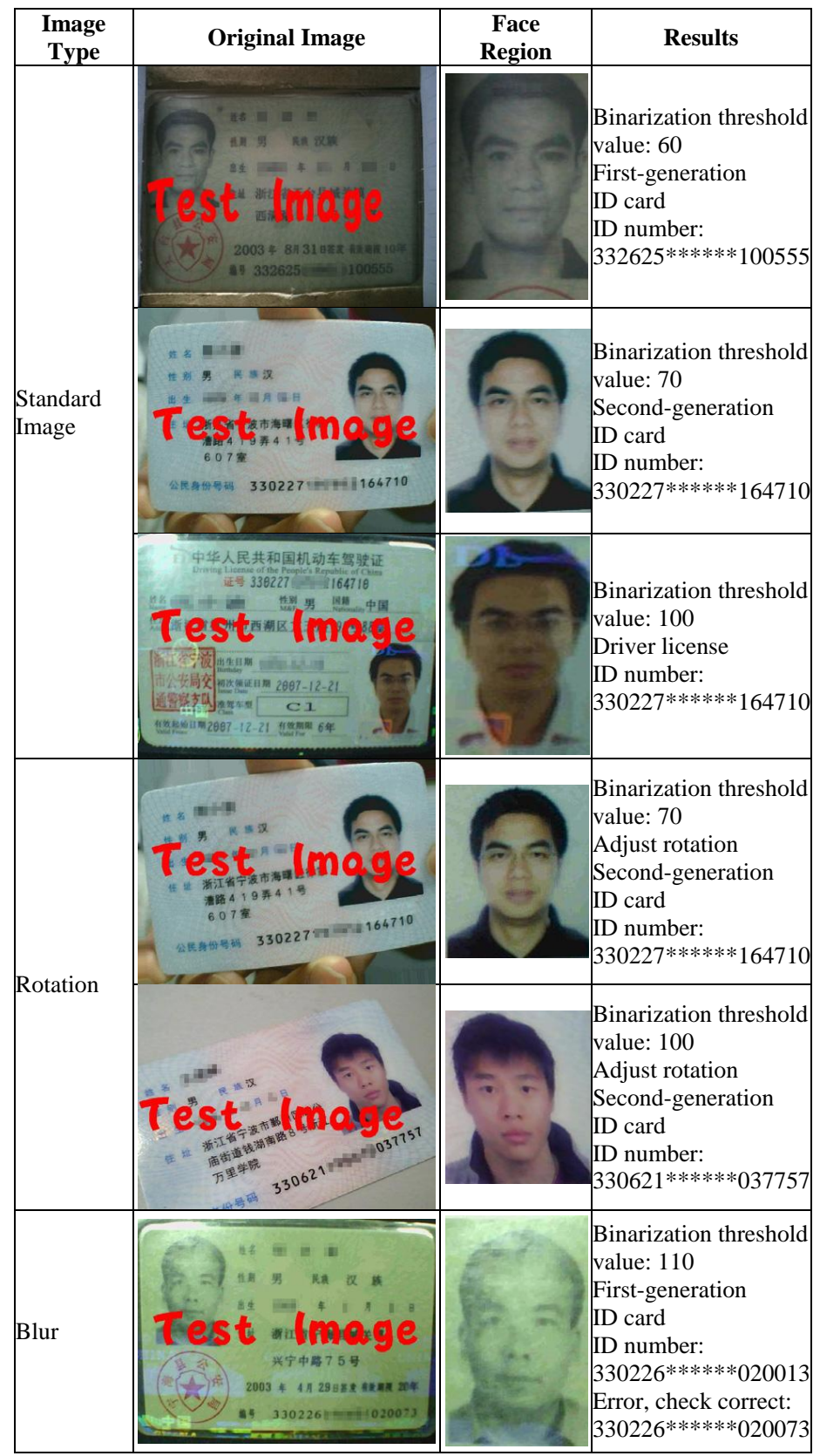




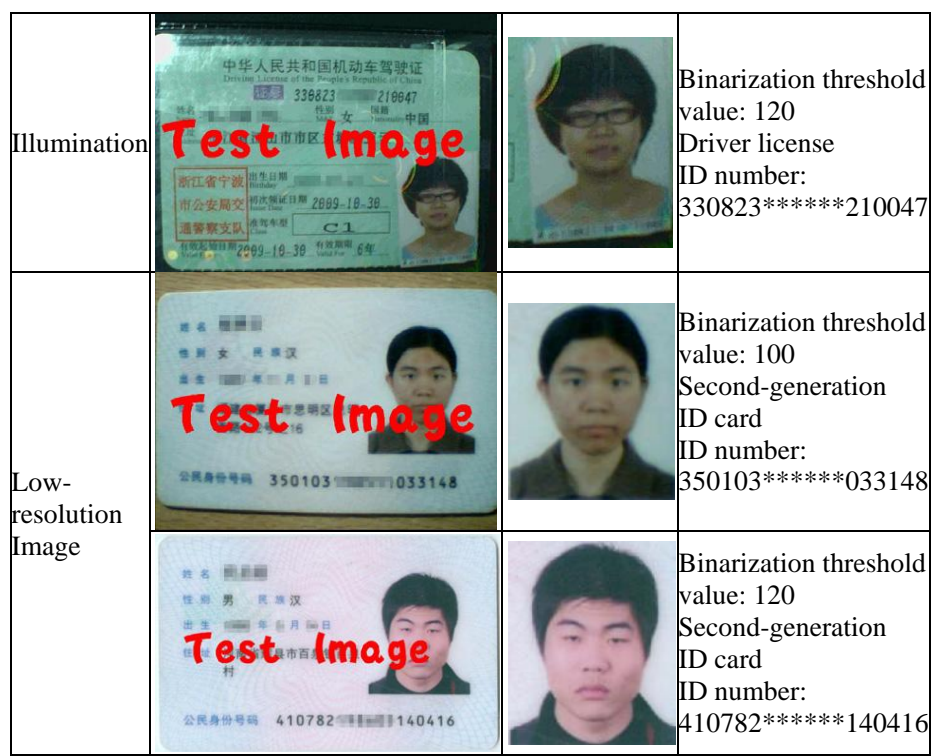

\section{CONCLUSIONS}

We have proposed a simple but efficient approach to recognize the ID numbers on three types of identification documents: the first generation ID card, the second-generation ID card and the driver license. We use the face features to determine the type of the ID card image, and we also use the face features to compute the rotation angle. In the stage of recognition, we use a novel method to vote the similarity about the unknown digital to each character template. Our approach is robust to the variations of illumination and rotation. The experimental results have demonstrated that the proposed approach is efficient, we tested a set of about 100 images which were shot by conventional digital cameras; for each image, the processing time is about one second on average, and the total recognition accuracy was up to $98 \%$; Our method reached higher recognition accuracy and processing speed than the current open source OCR engines. In the future, we will further study how to analyze the other information such as the text information automatically.

\section{REFERENCES}

[1] Suen, C.Y., et al. "Future Challenges in Handwriting and Computer Applications," 3rd International Symposium on Handwriting and Computer Applications, Montreal, May 29, 1987.

[2] Y. LeCun, B. Boser, J. S. Denker, D. Henderson, R. E. Howard, W. Hubbard, and L. D. Jackel. "Backpropagation applied to handwritten zip code recognition," Neural Computation, 1(4), pp. 541-551, Winter 1989.

[3] Y. LeCun, L. D. Jackel, B. Boser, J. S. Denker, H. P. Graf, I. Guyon, D. Henderson, R. E. Howard, and W. Hubbard. "Handwritten digit recognition: Applications of neural net chips and automatic learning," IEEE Communication, pp. 41-46, November 1989. invited paper.

[4] Tappert, Charles C., et al. "The State of the Art in On-line Handwriting Recognition," IEEE Transactions on Pattern Analysis and Machine Intelligence, vol. 12 No 8, August 1990, pp. 787-808.

[5] Y. LeCun, B. Boser, J. S. Denker, D. Henderson, R. E. Howard, W. Hubbard, and L. D. Jackel. Handwritten digit recognition with a backpropagation network. In David Touretzky, editor, Advances in Neural Information Processing Systems 2 (NIPS*89), Denver, CO, 1990. Morgan Kaufman.
[6] B. Boser, E. Sackinger, J. Bromley, Y. LeCun, and L. Jackel. "An analog neural network processor with programmable topology," IEEE Journal of Solid-State Circuits, 26(12), pp. 2017-2025, December 1991.

[7] Y. LeCun, L. D. Jackel, L. Bottou, A. Brunot, C. Cortes, J. S. Denker, H. Drucker, I. Guyon, U. A. Muller, E. Sackinger, P. Simard, and V. Vapnik. "Comparison of learning algorithms for handwritten digit recognition," In F. Fogelman and P. Gallinari, editors, International Conference on Artificial Neural Networks, pp. 53-60, Paris, 1995. EC2 $\&$ Cie.

[8] L. Bottou, Y. LeCun, and Y. Bengio. "Global training of document processing systems using graph transformer networks," In Proc. Of Computer Vision and Pattern Recognition, Puerto-Rico, 1997. IEEE.

[9] Y. LeCun, L. Bottou, and Y. Bengio. Reading checks with graph transformer networks. In International Conference on Acoustics, Speech, and Signal Processing, vol. 1, pp. 151-154, Munich, 1997. IEEE.

[10] Y. LeCun, L. Bottou, Y. Bengio, and P. Haffner. "Gradient-based learning applied to document recognition," Proceedings of the IEEE, november 1998.

[11] Nobuyuki Otsu. "A threshold selection method from gray-level histograms". IEEE Trans. Sys., Man., Cyber. 9, pp. 62-66.

[12] JIA Zhen-bin, TIAN Li-yan. "Recognition algorithm using BP neural network for ID," Journal of Suzhou Vocational University, 17(3):2006.

[13] Ruixiang Yin, Guohua Li. "An Automatic Recognition System for identifying ID Card Numbers," Journal of South China University of Technology (Natural Science Edition), 30(2):2002.

[14] Chi Ma, Hongyun Zhang, Duoqian Miao, Xuedong Zhang. "Improved Multi-threshold and Dynamic Binarization Algorithm," Computer Engineering, 32(06), pp. 203 205,2006.

[15] Ying Shen, Junbo Fan. "Algorithm Research of Character Location in ID Card Recogition," Computer Applications and Software, 21(03), pp. $80 \sim 82,2004$.

[16] Zhen Zhang, Shan Huang, Daizhang Li, Guoli Yan. "A Method Applied for Precise Segmentation of the Characters in the ID Card," Computer Engineering and Applications, 39(13):2003.

[17] Manhua Li, Ruixiang Yin, Chanhu Chen, "Preprocessing for ID Information Recognition," Journal of Shantou University (Natural Science Edition), 18(02):2003.

[18] http://code.google.com/p/tesseract-ocr.

\section{AUTHORS PROFILE}

Shen Lu, born in 1986, is currently a graduate at Xiamen University. He received his B.Sc. degree from Fuzhou University in 2009. His research interests largely lie in the areas of pattern recognition, machine learning, computer vision and related areas.

Yanyun Qu, received her B.Sc.and M.Sc. degrees in Computational Mathematics from Xiamen University and Fudan University, China, in 1995 and 1998, respectively, and received her Ph.D. degrees in Automatic Control from Xi'an Jiaotong University, China, in 2006. She joined the faculty of Department of Computer Science in Xiamen University since 1998. She was appointed as a lecturer from 2000 to 2007 and was appointed as an associate professor since 2007. She is a member of the IEEE. Her current research interests include pattern recognition, computer vision, image/video processing, machine learning, etc.

Yanyun Cheng, born in 1985, is currently a graduate at Xiamen University. She was awarded a B.Sc. in Computer Science and Technology Department, Xiamen University in 2008. Her research interests largely lie in the areas of pattern recognition, machine learning, computer vision and related areas.

Yi Xie, received her B.Sc. and M.Sc. degrees from Xi'an Jiaotong University and received her Ph.D. degree from The Hong Kong Polytechnic University in 2008. Currently, she is an assistant professor with Department of Computer Science in Xiamen University. Her current research interests include image/video processing, system modeling, system simulation and network protocol analysis. 
APPEXDIX

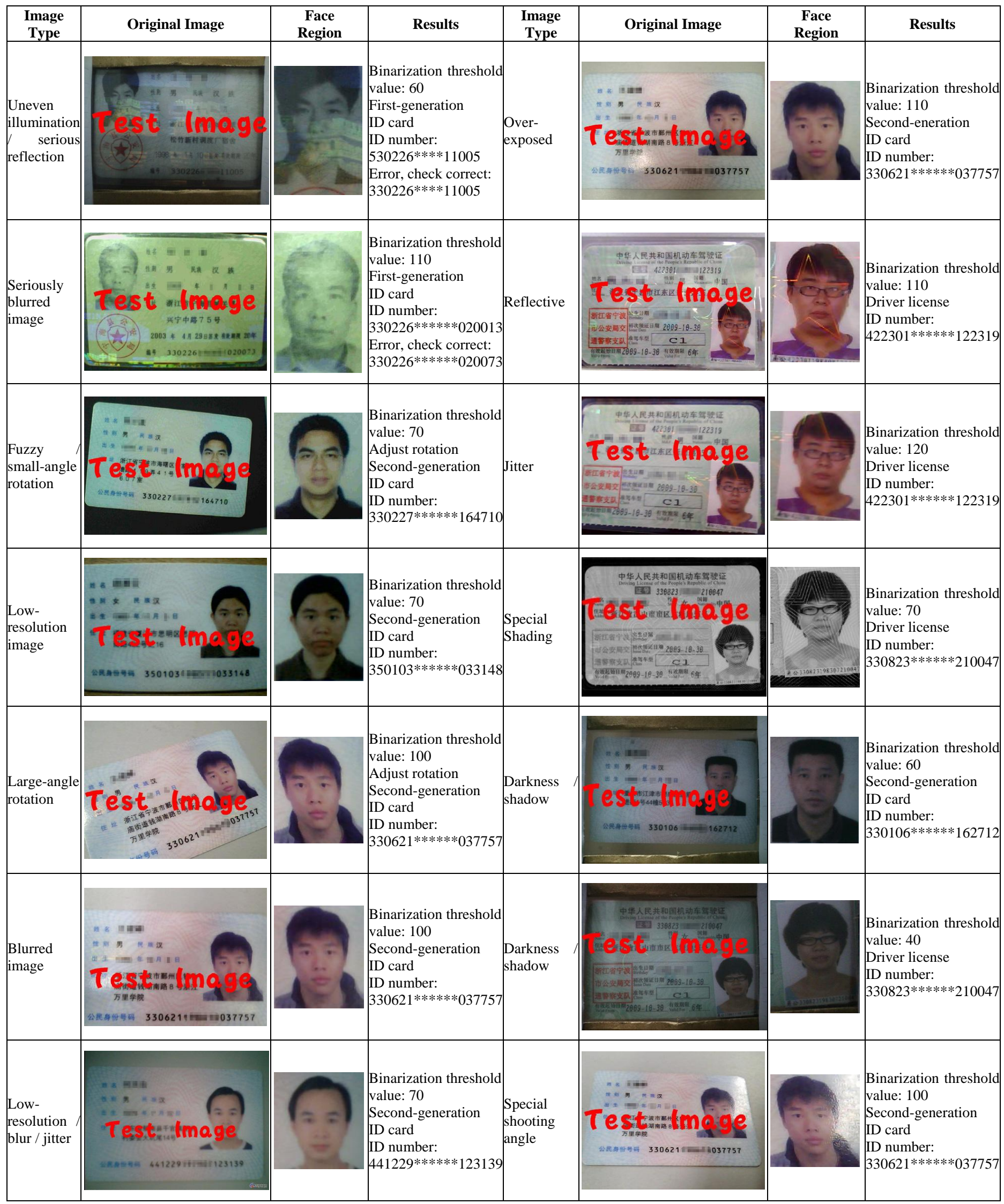


(IJACSA) International Journal of Advanced Computer Science and Applications, Special Issue on Image Processing and Analysis

\begin{tabular}{|c|c|c|c|c|c|}
\hline$\left|\begin{array}{lr}\text { A } & \text { complex } \\
\text { background } \\
\text { in } & \text { larger } \\
\text { proportion }\end{array}\right|$ & - & $\begin{array}{l}\text { Binarization threshold } \\
\text { value: } 110 \\
\text { Second-generation } \\
\text { ID card } \\
\text { ID number: } \\
232103 * * * * * 295819\end{array}$ & $\begin{array}{l}\text { Low- } \\
\text { resolution / } \\
\text { small-angle } \\
\text { rotation }\end{array}$ & 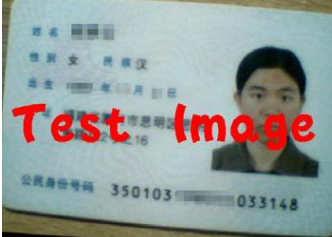 & $\begin{array}{l}\text { Binarization threshold } \\
\text { value: } 100 \\
\text { Adjust rotation } \\
\text { Second-generation } \\
\text { ID card } \\
\text { ID number: } \\
350103 * * * * * * 033148\end{array}$ \\
\hline
\end{tabular}

